\title{
FIBONACCI AND TELEPHONE NUMBERS IN EXTREMAL TREES
}

\author{
Urszula Bednarz AND IwONa WŁOCH \\ Faculty of Mathematics and Applied Physics \\ Rzeszow University of Technology \\ Powstańców Warszawy 12, 35-359 Rzeszów, Poland \\ e-mail: ubednarz@prz.edu.pl \\ iwloch@prz.edu.pl
}

\begin{abstract}
In this paper we shall show applications of the Fibonacci numbers in edgecoloured trees. In particular we determine the successive extremal graphs in the class of trees with respect to the number of $(A, 2 B)$-edge colourings. We show connections between these numbers and Fibonacci numbers as well as the telephone numbers.
\end{abstract}

Keywords: edge colouring, tripod, Fibonacci numbers, telephone numbers.

2010 Mathematics Subject Classification: 11B37, 11C20, 15B36, 05C69.

\section{REFERENCES}

[1] U. Bednarz, I. Włoch and M. Wołowiec-Musiał, Total graph interpretation of numbers of the Fibonacci type, J. Appl. Math. (2015) ID 837917. 
[2] U. Bednarz, D. Bród, A. Szynal-Liana, I. Włoch and M. Wołowiec-Musiał, On Fibonacci numbers in edge coloured trees, Opuscula Math. 37 (2017) 479-490.

[3] R. Diestel, Graph Theory (Springer-Verlag, Heidelberg, New York, Inc., 2005).

[4] I. Gutman and S. Wagner, Maxima and minima of the Hosoya index and the Merrifield-Simmons index. A survey of results and techniques, Acta Appl. Math. 112 (2010) 323-346.

[5] H. Prodinger and R.F. Tichy, Fibonacci numbers of graphs, Fibonacci Quart. 20 (1982) 16-21.

[6] J. Riordan, Introduction to Combinatorial Analysis (Dover, 2002) 85-86.

[7] R.F. Tichy and S. Wagner, Extremal problems for topological indices in combinatorial chemistry, J. Comput. Biol. 12 (2005) 1004-1013.

[8] E. Weisstein, Tripod index entries for linear recurrence with constant coefficients, MathWorld, Wolfram Web Resource, Mar 05 (2011). http://mathworld.wolfram.com/Tripod.html

[9] The On-Line Encyclopedia of Integer Sequences. https://oeis.org/

Received 19 April 2016

Revised 18 October 2016 Accepted 18 October 2016 